\title{
Structure of potassium cyanide at low temperature and high pressure determined by neutron diffraction
}

\author{
Harold T. Stokes, Daniel L. Decker, and H. Mark Nelson \\ Department of Physics and Astronomy, Brigham Young University, Provo, Utah 84602 \\ J. D. Jorgensen \\ Materials Science Division, Argonne National Laboratory, Argonne, Illinois 60439
}

(Received 14 December 1992)

\begin{abstract}
Neutron time-of-flight powder-diffraction spectra have been obtained in potassium cyanide (KCN) at temperatures between 25 and $200 \mathrm{~K}$ and at pressures between 35 and $366 \mathrm{MPa}$. These data sample five different phases of $\mathrm{KCN}$. In phase $C$, which exists at $366 \mathrm{MPa}$ and below $170 \mathrm{~K}$, the structure is determined to be paraelectric with the monoclinic space-group symmetry $C 2 / c$. This is in disagreement with a previous study which determined the structure to be ferroelectric with space-group symmetry $C c$. In phase $D$, which exists at $366 \mathrm{MPa}$ and below $120 \mathrm{~K}$, the structure is determined to be paraelectric with the monoclinic space-group symmetry $C 2 / \mathrm{m}$. The cyanide ions lie in mirror planes. In both phases $C$ and $D$, the cyanide ions are ordered with respect to the direction of their bonds but disordered with respect to interchange of $\mathrm{C}$ and $\mathrm{N}$. Also in these phases, line broadening due to an inhomogeneous shear strain was observed. A mathematical treatment of this phenomenon is given.
\end{abstract}

\section{INTRODUCTION}

Potassium cyanide $(\mathrm{KCN})$ has been the subject of numerous structural studies. In Fig. 1 is shown the currently known phase diagram of $\mathrm{KCN}$. Phase I has the cubic $\mathrm{NaCl}$ structure (space group $F m \overline{3} m$ ). The structure of phase $A$ was established by Bijvoet and Lely ${ }^{1}$ to be orthorhombic (space group Immm). The structures of phases III and IV were determined by Decker et al. ${ }^{2}$ to be cubic ( $\mathrm{CsCl}$ structure, space group $P m \overline{3} m$ ) and monoclinic (space group $C m$ ), respectively. Phase $B$ was determined by Rowe, Rush, and Prince ${ }^{3}$ to have an antiferroelectric structure (space group $P m m n$ ). Dultz et $a l^{4}$ studied phases $C$ and $D$ by neutron diffraction. They found both phases to be monoclinic. They fit their data in phase $C$ to space group $C c$, but they were unable to determine the structure of phase $D$.

The phases in KCN differ from each other not only in positions of atoms, but also in the dynamic disorder of the $\mathrm{CN}^{-}$ions. In phases $\mathrm{I}$ and III, the $\mathrm{CN}^{-}$ions are reorienting with almost spherical symmetry. In phase $A$, the $\mathrm{CN}^{-}$ions are partially ordered: the $\mathrm{CN}^{-}$bonds line up along a common direction but the $\mathrm{CN}^{-}$ions still reorient randomly head to tail. In phases $B$ and IV, the $\mathrm{CN}^{-}$ions are ordered with respect to $\mathrm{C}$ and $\mathrm{N}$ : these phases are antiferroelectric and ferroelectric, respectively. Dultz et $a l .{ }^{4}$ reported that in phase $C$ the $\mathrm{CN}^{-}$ions are ordered in a ferroelectric structure. The ordering of the $\mathrm{CN}^{-}$ions in phase $D$ has not been previously determined.

We obtained neutron-diffraction spectra from powdered samples of $\mathrm{KCN}$ under high pressure at low temperatures. We obtained spectra in phases I, $A, B, C$, and $D$. In this paper we report the analysis of these spectra.

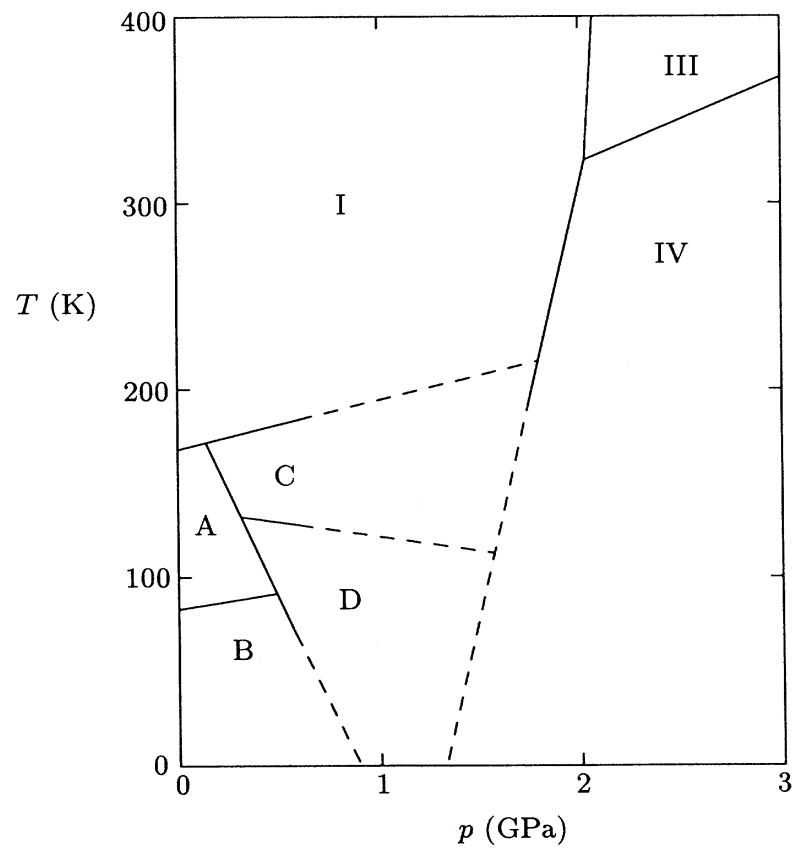

FIG. 1. Phase diagram of KCN. The solids lines represent experimentally determined phase boundaries from Refs. 2 and 15. The dashed lines are extrapolations. The hysteresis of the phase boundaries around phases $C$ and $D$ is not shown. 
We have determined the structure of phases $C$ and $D$. Our result for phase $C$ disagrees with that of Dultz et al. ${ }^{4}$

\section{EXPERIMENTAL METHODS}

Neutron-powder-diffraction data were taken using the Special Environment Powder Diffractometer (SEPD) at the Intense Pulsed Neutron Source (IPNS), a facility of the Argonne National Laboratory. ${ }^{5}$ This is a time-offlight (TOF) instrument, allowing full diffraction data to be collected at a single scattering angle. For highpressure experiments, a scattering angle of $2 \theta=90^{\circ}$ is chosen to allow scattering from the pressure cell to be eliminated through appropriate collimation.

The sample was placed in an aluminum-alloy cell capable of $400 \mathrm{MPa}$ pressure (described in Ref. 6). The sample chamber was cylindrical, $1.2 \mathrm{~cm}$ in diameter and $5 \mathrm{~cm}$ long. The pressure cell was equipped with cadmium collimators to allow for neutrons scattered from a sample to be detected while masking the scattering from the pressure cell walls. Pressure was applied through a small capillary tube from a $\mathrm{He}$ gas intensifier and measured using a Bourdon gauge and was stable to about \pm 2 $\mathrm{MPa}$.

The pressure cell was cooled by a Displex closed-cycle helium refrigerator. The temperature was measured by a thermocouple on the displex cold plate. The temperature was accurate to about $\pm 1 \mathrm{~K}$.

The data sets obtained are listed in Table I in chrono- logical order. They are also shown as points on Fig. 2. Between data sets, the pressure in the sample chamber was first changed to the desired final value, and then the temperature.

Each data set obtained at a particular pressure $p$ and temperature $T$ required about $6 \mathrm{~h}$ of data collecting and contained over 600000 neutron counts distributed over flight times of $1.5-30 \mathrm{~ms}$, corresponding to plane spacings between 0.27 and $5.4 \AA$. In Fig. 3 is shown a representative neutron-diffraction spectra for each phase.

Two different samples were used. Sample 1 was obtained from Matthew DeLong (University of Utah). It was a single crystal which we ground in a dry box. Sample 2 was obtained from David Hinks (MSD, Argonne). It was a high-purity powder which had been stored in dry $\mathrm{N}_{2}$ gas. Data sets 1-14 were taken using sample 1, and data sets 15-19 were taken using sample 2. Sample 1 showed preferential line broadening in the high pressure phases, possibly due to strains introduced on grinding. Sample 2 also showed some of the same line broadening, but to a much lesser degree.

Each data set was analyzed by the Reitveld structural refinement technique using a computer program TOFLS. ${ }^{7}$ The goodness of fit was judged from the value of $R_{\mathrm{wp}} / R_{\text {exp }}$, the ratio of the weighted profile $R$ value to the expected $R$ value. TOFLS used an incident neutron intensity profile which was obtained by measuring the transmitted neutron beam after it passes through the cell.

TOFLS also allowed a two-parameter fit to the background neutrons. After allowing the program to subtract

TABLE I. Data sets.

\begin{tabular}{crrrllll}
\hline \hline Data set & $p(\mathrm{MPa})$ & $T(\mathrm{~K})$ & Phase & $a(\AA)$ & $b(\AA)$ & $c(\AA)$ & $\beta(\mathrm{deg})$ \\
\hline Sample 1 & & & & & & & \\
1 & 364 & 45 & B & $6.0288(3)$ & $4.1523(2)$ & $5.2341(4)$ & \\
2 & 255 & 45 & $B$ & $6.0429(2)$ & $4.1633(2)$ & $5.2377(3)$ & \\
3 & 145 & 45 & $B$ & $6.0581(2)$ & $4.1750(2)$ & $5.2414(3)$ & \\
4 & 35 & 45 & $B$ & $6.0735(2)$ & $4.1870(2)$ & $5.2457(3)$ & \\
5 & 46 & 120 & $A$ & $6.1342(2)$ & $4.2240(1)$ & $5.1842(2)$ & \\
6 & 54 & 200 & I & $6.4802(1)$ & & & \\
7 & 366 & 200 & I & $6.4370(1)$ & & & \\
8 & 365 & 150 & $C$ & $7.441(1)$ & $4.595(1)$ & $9.115(1)$ & $122.14(1)$ \\
9 & 365 & 110 & $D$ & $7.932(1)$ & $4.274(1)$ & $9.460(1)$ & $125.21(1)$ \\
10 & 365 & 98 & $D$ & $7.935(1)$ & $4.268(1)$ & $9.465(1)$ & $125.24(1)$
\end{tabular}




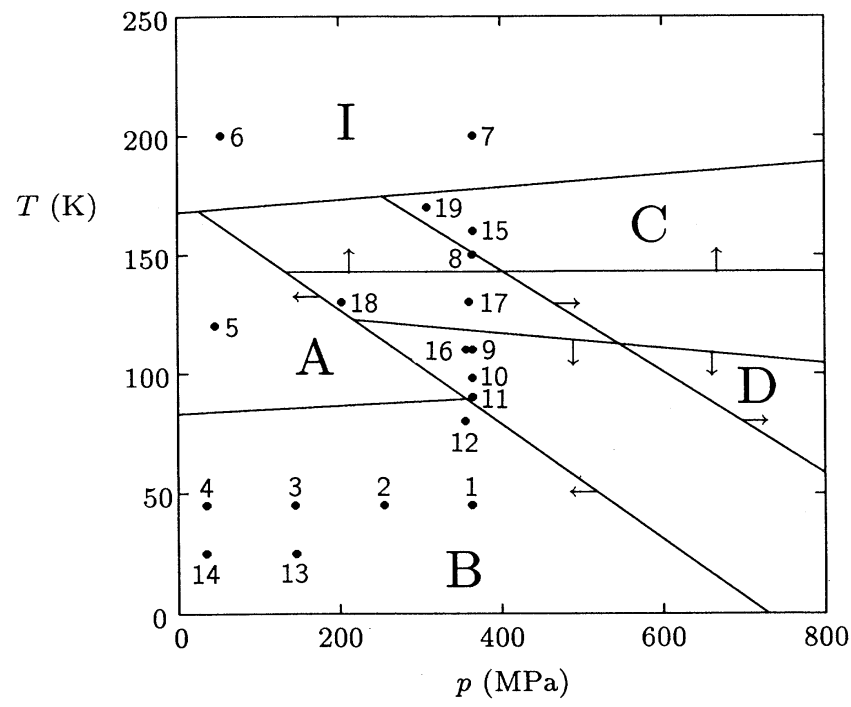

FIG. 2. Phase diagram of KCN. The lines separating the phases are from Ref. 15. The arrows indicate hysteresis in the phase transitions. For example, the line with arrows pointing vertically upward indicates the phase transition from phase $D$ to $C$ with increasing temperature. The line with arrows pointing vertically downward indicates the phase transition from phase $C$ to $D$ with decreasing temperature. The numbered points on the diagram indicate the various data sets listed in Table I.

out the background from each data set the best it could, we then subtracted the small amount of remaining background "by hand." After this point, we did not allow the program to further adjust the background parameters. Figure 3 shows the spectra after the background had been subtracted out. We note that the diffuse scattering contribution to the background was so strong that we could not extend the analysis of Bragg data to $d$ values smaller than about $1.3 \AA$. We analyzed only the range of $d$ values shown in Fig. 3 .

TOFLS models atomic motion with thermal ellipsoids and assumes that the motion is harmonic. This is not a good model for the motion of the $\mathrm{C}$ and $\mathrm{N}$ atoms. The $\mathrm{C}-\mathrm{N}$ bond causes the atomic motion to be rather more complicated. Therefore, we constrained the thermal ellipsoids of the $\mathrm{C}$ and $\mathrm{N}$ atoms to be equal. Allowing different thermal ellipsoids for the $\mathrm{C}$ and $\mathrm{N}$ atoms would unnecessarily increase the number of adjustable parameters in a model that is only an approximation to physical reality in the present case. Furthermore, in phases $C$ and $D$, we used isotropic thermal parameters for all atoms, in order to keep the number of adjustable parameters to a manageable number.

\section{PHASE I}

In phase $\mathrm{I}, \mathrm{KCN}$ has the cubic $\mathrm{NaCl}$ structure: space group No. $225 \mathrm{Fm} \overline{3} m\left(O_{h}^{5}\right)$. The $\mathrm{K}^{+}$ions occupy the Wyckoff $4 b$ position $\left(\frac{1}{2}, \frac{1}{2}, \frac{1}{2}\right)$. The $\mathrm{CN}^{-}$ions undergo rapid reorientations centered at the Wyckoff $4 a$ position $(0,0,0)$. The time-averaged symmetry of the $\mathrm{CN}^{-}$ions about that point is cubic (point group $m \overline{3} m$ ). The rotation is not free, but is hindered. The potential energy of the $\mathrm{CN}^{-}$ion is lower for the $\langle 111\rangle$ directions of orientation, ${ }^{8}$ and the $\mathrm{CN}^{-}$ion spends more time near those minima.

Data sets 6 and 7 were both taken at $200 \mathrm{~K}$, but at two different pressures. From our fit to these data sets, we obtained the lattice parameters $a$ shown in Table I. From these results, we obtain a bulk modulus equal to 15.5(2) GPa for phase I.

\section{PHASE $A$}

The phase transition from phase I to $A$ involves a partial ordering of the $\mathrm{CN}^{-}$ions. In phase I, the $\mathrm{CN}^{-}$ions reorient rapidly in all directions. Their time-averaged symmetry is $m \overline{3} m$ (nearly spherical). At the transition to phase $A$, the $\mathrm{CN}^{-}$ions line up along the cubic [110] direction, but still reorient rapidly head to tail. Their timeaveraged symmetry is now $\mathrm{mmm}$ (nearly cigar shaped). The resulting crystal symmetry is orthorhombic: space group No. $71 \mathrm{Immm}\left(D_{2 h}^{25}\right) .{ }^{1}$ The $\mathrm{K}^{+}$ions occupy the Wyckoff $2 a$ position $(0,0,0)$, and the $\mathrm{CN}^{-}$ions are centered at the Wyckoff $2 b$ position $\left(0, \frac{1}{2}, \frac{1}{2}\right)$. The C-N bonds point in the direction of the orthorhombic $c$ axis.

In Fig. 4(a) we show the structure of phase I. The small

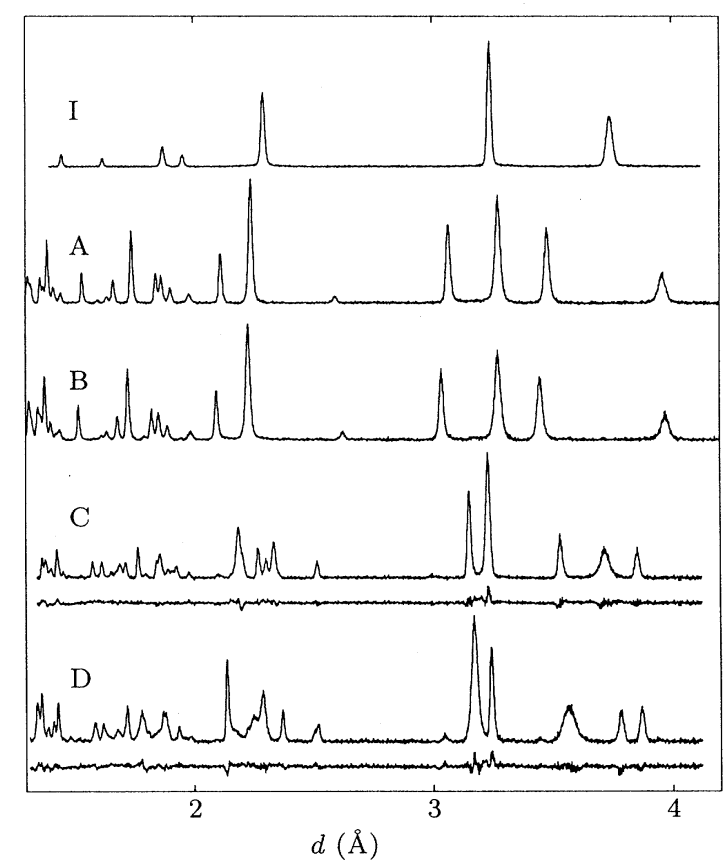

FIG. 3. A representative neutron-diffraction spectrum for each phase. The background has been subtracted from the raw data. Also, the number of neutron counts at each $d$ value has been divided by the square root of the incident neutron intensity at that $d$ value, so that each spectrum has a constant noise level across its entire width. The spectra shown for phases I, $A, B, C, D$ are taken from data sets $6,5,4,15,16$, respectively. Below the spectra for phases $C$ and $D$ are shown the difference between the data and the calculated spectra from our best fit. 
circles represent $\mathrm{K}^{+}$ions, and the large circles represent the rapidly reorienting $\mathrm{CN}^{-}$ions. The plane of the figure is parallel to the (110) cubic plane. The blank circles lie in the plane of the figure, and the circles with crosses in them lie in a plane $\frac{1}{4} \sqrt{2} a$ above the plane of the figure. In Fig. 4(b) we show the ordering of the $\mathrm{CN}^{-}$ions in phase $A$. The pairs of large circles represent the $\mathrm{CN}^{-}$ ions which are now reorienting only head to tail.

The lattice parameters $a, b, c$ obtained from the fits to the neutron spectra in phase $A$ are shown in Table I. In data sets $12,16,17,18$, we could identify diffraction lines from both phase $D$ and from phase $A$ or $B$. (For example, in Fig. 3 the 110 line of phase $A$ is clearly seen at $d=3.45 \AA$ in the spectrum for phase $D$.) These data sets contain a mixture of two phases. The percentage of each phase present is given in Table I. These percentages are estimated from the relative intensities of the diffraction peaks in the data. Since the spectra for phases $A$ and $B$ are so similar, we could not distinguish between them in these data sets. We assigned phase $B$ to data set 12 and phase $A$ to data sets $16,17,18$ simply on the basis of their positions on the phase diagram in Fig. 2. In data sets 16 and 17, we could not reliably determine the lat-
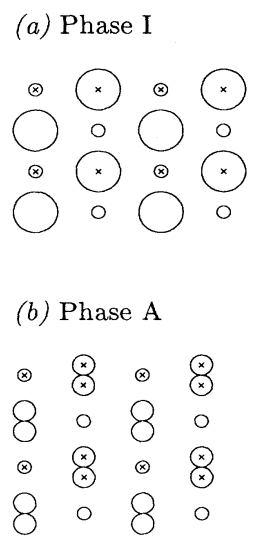

(c) Phase B

$\begin{array}{llll}\otimes & \otimes & \otimes & \otimes \\ 8 & 0 & 8 & 0 \\ \otimes & \otimes & \otimes & \otimes \\ 8 & 0 & 8 & 0\end{array}$

FIG. 4. The structure of phases I, $A, B$. The plane of the figure is parallel to the cubic (110) plane in phase I and the orthorhombic (101) plane in phases $A$ and $B$. The blank circles lie in the plane of the figure, and the circles with crosses in them lie in a plane approximately $2.3 \AA\left(\frac{1}{4} \sqrt{2} a\right)$ above the the plane of the figure. The small circles represent $\mathrm{K}^{+}$ions, and the large circles represent $\mathrm{CN}^{-}$ions. In phase $\mathrm{I}$, the $\mathrm{CN}^{-}$ ions are reorienting in all directions and are each represented by one large circle. In phase $A$, the $\mathrm{CN}^{-}$ions are aligned but still reorienting head to tail and are each represented by two circles of equal diameter. In phase $B$, the $\mathrm{CN}^{-}$ions are ordered with respect to $\mathrm{C}$ and $\mathrm{N}$, and are each represented by a pair of circles, one larger ( $\mathrm{C}$ atom) and one smaller ( $\mathrm{N}$ atom). tice parameters $a, b, c$ of phase $A$ because the peaks were so small and only a couple of them could be definitely identified.

\section{PHASE $B$}

The phase transition from phase $A$ to $B$ is orderdisorder. In phase $A$ the $\mathrm{C}-\mathrm{N}$ bonds all point in the direction of the orthorhombic $c$ axis, but the $\mathrm{CN}^{-}$ions rapidly reorient head to tail. In phase $B$, the $\mathrm{CN}^{-}$ions become ordered with respect to $\mathrm{C}$ and $\mathrm{N}$. Half of the $\mathrm{CN}^{-}$ions point in the orthorhombic $+c$ direction, and the other half point in the $-c$ direction. This phase is antiferroelectric. The crystal symmetry is still orthorhombic: space group No. $59 P m m n\left(D_{2 h}^{13}\right){ }^{3}$ The $\mathrm{K}^{+}$ions occupy the Wyckoff $2 a$ position $\left(\frac{1}{4}, \frac{1}{4}, z\right)$, and the $\mathrm{C}$ and $\mathrm{N}$ atoms occupy the Wyckoff $2 b$ position $\left(\frac{1}{4}, \frac{3}{4}, z\right)$. We use the second setting of Pmmn in International Tables for Crystallography, ${ }^{9}$ where the point of inversion is at the origin. The structure of phase $B$ is shown in Fig. $4(\mathrm{c})$.

As can be seen in Fig. 3, the diffraction spectra observed for phases $A$ and $B$ are nearly identical. The ordering of the $\mathrm{CN}^{-}$ions causes very little change in the spectrum. In Fig. 5 we show a portion of the spectrum in phase $B$. Two weak lines are labeled: 212 and 021 . These lines are forbidden by symmetry in phase $A$. They constitute the only firm evidence from our neutron-diffraction data that a lowering of symmetry does indeed take place in the transition from phase $A$ to $B$.

The lattice parameters resulting from our fits to the neutron-diffraction spectra in phase $B$ are given in Table I. The atomic position parameters $z$ for the K,C,N atoms are $0.732(2), 0.141(1)$, and $0.360(1)$, respectively (nearly the same for every data set).

Data sets 1-4 were taken at the same temperature (45 K) but at different pressures. From these four data sets we calculate the strain along each of the three axes. The result is shown in Fig. 6. The lines in the figure are linear

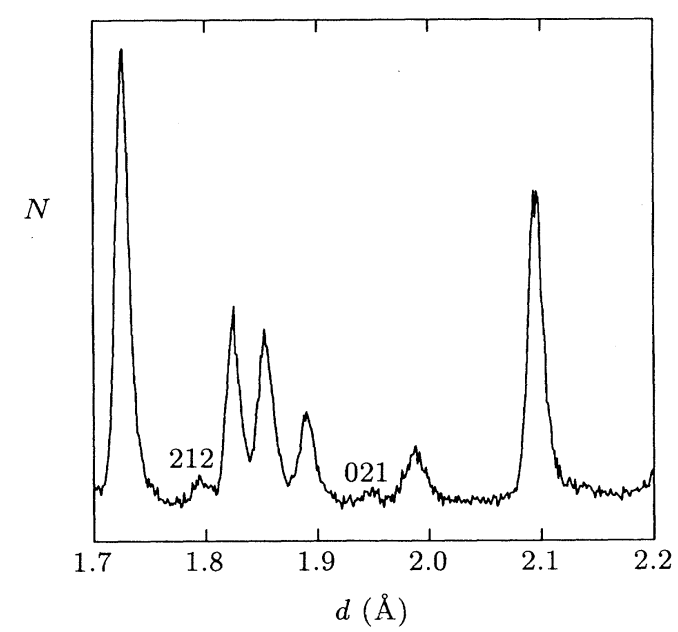

FIG. 5. Details of the neutron-diffraction spectrum for phase $B$, data set 4 , showing two lines, 212 and 021 , which are forbidden in phase $A$. 


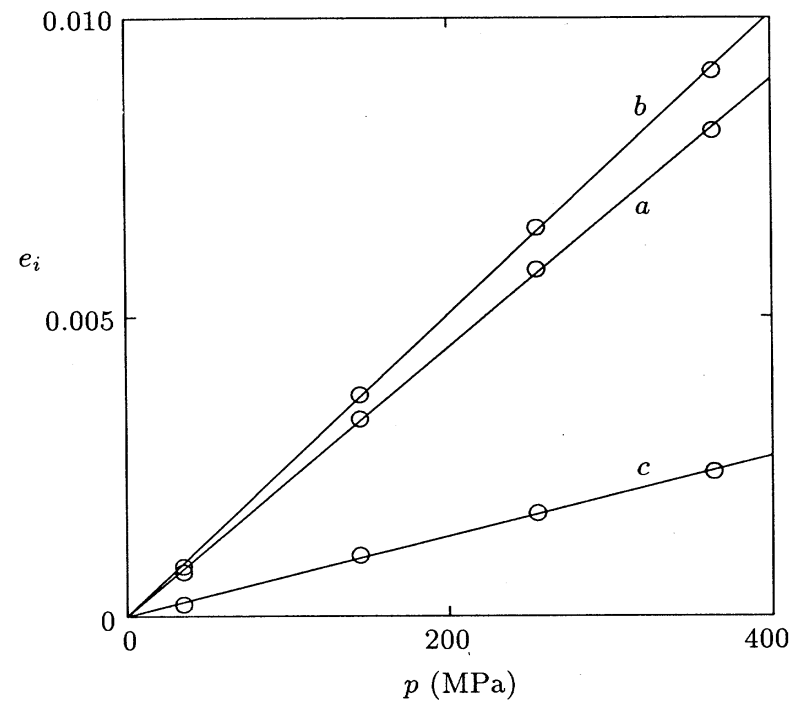

FIG. 6. Strain along the $a, b, c$ directions as a function of pressure in phase $B$ at $45 \mathrm{~K}$.

least-squares fits to the data points. The values of strain are adjusted so that each line passes through the origin in the figure. The strain per unit pressure is the slope of the line.

In terms of the elastic compliance constants $S_{i j}$, the strain per unit pressure is given by the expression,

$$
e_{i}=\sum_{j=1}^{3} S_{i j}
$$

These constants were calculated by Stokes and Hatch ${ }^{10}$ from a microscopic model of $\mathrm{KCN}$ using interatomic forces of the Born-Mayer type. In Table II, we compare the strains along the three axes calculated from their results with our experimentally determined values. The differences are most likely due to the inadequacy of the model used in the calculations.

Data sets 13 and 14 were taken at $25 \mathrm{~K}$. The relative strains observed at that temperature are nearly the same as those observed for data sets 3 and 4 at $45 \mathrm{~K}$. We also note from Table I that the coefficient of linear thermal expansion along the $c$ axis is negative.

TABLE II. Strain $e_{i}$ per unit pressure $p$ along each of the three orthorhombic axes $a, b, c$ in phase $B$. Our experimentally determined values are compared with values calculated by Stokes and Hatch (Ref. 10).

\begin{tabular}{lll}
\hline \hline Axis & $e_{i} / p\left(\mathrm{~Pa}^{-1}\right)$ & \\
& Expt. & Calc. \\
\hline$a$ & $2.24(1) \times 10^{-11}$ & $3.4 \times 10^{-11}$ \\
$b$ & $2.51(2) \times 10^{-11}$ & $3.2 \times 10^{-11}$ \\
$c$ & $0.67(2) \times 10^{-11}$ & $1.0 \times 10^{-11}$ \\
\hline
\end{tabular}

PHASE $C$

We have determined from our data that phase $C$ is monoclinic, space group No. $15 C 2 / c\left(C_{2 h}^{6}\right)$. The directions of the $\mathrm{C}-\mathrm{N}$ bonds are ordered, but the $\mathrm{CN}^{-}$ions are disordered with respect to $\mathrm{C}$ and $\mathrm{N}$ (like phase $A$ ). The $\mathrm{K}$ atoms are at the Wyckoff position $4 e\left(0, y, \frac{1}{4}\right)$, and the $\mathrm{CN}^{-}$ions are centered at the Wyckoff position $4 a(0,0,0)$. The atomic positions are shown in Table III. (We use the setting of unique axis $b$ and cell choice 1 in the International Tables for Crystallography. ${ }^{9}$ ) The structure of phase $C$ is shown in Fig. 7. The C-N bonds point out of the plane of the figure, the angle between the $\mathrm{C}-\mathrm{N}$ bonds and the plane of the figure being approximately $45^{\circ}$.

Some of the diffraction peaks in the data for phase $C$ are very broad. This is probably due to inhomogeneous strains in the sample. Similar broadening was observed in $\mathrm{RbCN}{ }^{11}$ The broadening was much greater in sample 1 than in sample 2. In Fig. 3 is shown the spectrum for phase $C$ obtained from sample 2 (data set 15). In Fig. 8 we show a spectrum for phase $C$ from sample 1 (data set 8). If we compare these two spectra, we see that some lines are more affected by the inhomogeneous strain than others. We find that lines such as 110,112 , and $31 \overline{2}$ are very much affected by the strain, and lines such as $11 \overline{3}$, $40 \overline{4}, 11 \overline{5}$, and 400 are affected very little by the strain.

From this information, we determined that the strain

TABLE III. Structures of phase $C$ in KCN and a similar phase in $\mathrm{RbCN}$. In the first column is our $C 2 / c$ structure of $\mathrm{KCN}$. In the second column is the $C c \mathrm{KCN}$ structure of Dultz et al (Ref. 4). We use a space-group setting for their data which would place the atoms very near the positions of the atoms in our $C 2 / c$ structure. We transformed their reported positions of the $\mathrm{K}$ atoms according to $z,-y+\frac{1}{2}, x+\frac{1}{4}$ and the positions of the $\mathrm{C}$ and $\mathrm{N}$ atoms according to $z-\frac{1}{2}, y, x-\frac{1}{4}$. In the third column is the $C c \mathrm{RbCN}$ structure of Rowe, Rush, and Lüty (Ref. 11). As with the second column, we again use a space-group setting which would place the atoms very near the positions of the atoms in our $C 2 / c$ structure of $\mathrm{KCN}$. We transformed the reported positions of their $\mathrm{Rb}$ atoms according to $x, y+\frac{1}{2}, z+\frac{1}{4}$ and the positions of their $\mathrm{C}$ and $\mathrm{N}$ atoms according to $x-\frac{1}{2},-y, z-\frac{1}{4}$.

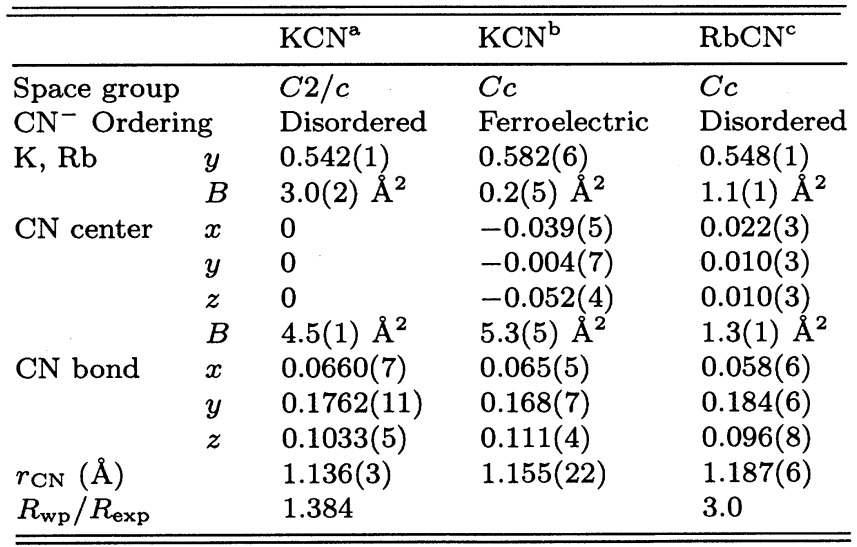

aThis work.

${ }^{\mathrm{b}}$ Reference 4 .

${ }^{\mathrm{c}}$ Reference 11 . 


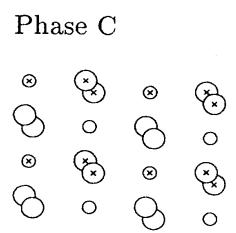

FIG. 7. The structure of phase $C$. These are the same set of atoms shown in Fig. 4 for the cubic and orthorhomic phases. The plane of this figure is parallel to the monoclinic (010) plane, and the monoclinic $c$ axis points vertically upward on the page. The symbols in the figure have the same meaning as in Fig. 4.

is very nearly a pure shear strain. In the case of a shear strain, one can always choose three mutually perpendicular vectors, $\hat{\mathbf{n}}_{1}, \hat{\mathbf{n}}_{2}, \hat{\mathbf{n}}_{3}$, such that the strain changes the angle between $\hat{\mathbf{n}}_{1}$ and $\hat{\mathbf{n}}_{2}$ but does not change the angle between $\hat{\mathbf{n}}_{1}$ and $\hat{\mathbf{n}}_{3}$ or between $\hat{\mathbf{n}}_{2}$ and $\hat{\mathbf{n}}_{3}$. The two vectors, $\hat{\mathbf{n}}_{1}$ and $\hat{\mathbf{n}}_{2}$, represent the "direction" of the shear strain. In our samples, we obtained a best fit to the data when $\hat{\mathbf{n}}_{1}$ and $\hat{\mathbf{n}}_{2}$ are pointing in the directions $\mathbf{b}$ and $\mathbf{a}+0.27 \mathbf{c}$, respectively.

Note that this strain destroys the monoclinic symmetry. The $b$ axis is no longer perpendicular to the $a c$ plane. The inhomogeneous shear strain tilts the $b$ axis in one direction in some parts of the sample, and in the opposite direction in other parts of the sample. Only on the average is the symmetry of the crystal monoclinic.

For the case of a shear strain, the broadening of diffraction lines is described by the expression (see the Appendix),

$$
\Delta d_{h k l}=C d \cos \phi_{1} \cos \phi_{2},
$$

where $\phi_{i}$ is the angle between $\hat{\mathbf{n}}_{i}$ and the direction normal to the $(h k l)$ plane. To fit our data, we modified TofLs to use this expression as a contribution to the total width of each line. In data set 15 , for example, this resulted in an improvement in the value of $R_{\mathrm{wp}} / R_{\exp }$ from 2.069 to 1.384. In Fig. 3, we plot the difference between the data and the spectrum calculated from our best fit.

Dultz et al. ${ }^{4}$ determined phase $C$ to be ferroelectric, space group No. $9 C c\left(C_{s}^{4}\right)$, in disagreement with our result. In their structure, the $\mathrm{CN}^{-}$ions are completely ordered with respect to $\mathrm{C}$ and $\mathrm{N}$. We give in Table III the atomic positions they obtained for their structure. We see that except for the ordering of the $\mathrm{CN}^{-}$ions,

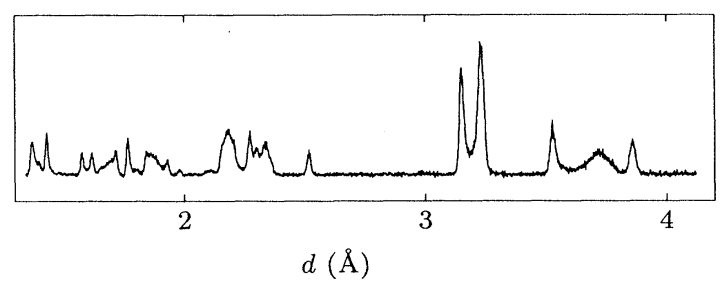

FIG. 8. The neutron-diffraction spectrum for phase $C$ in sample 1 (data set 8). Some of the lines are broader than in sample 2 (see Fig. 3) because of a greater amount of inhomogeneous strain in sample 1. their $C c$ structure is very similar to our $C 2 / c$ structure, especially in the orientation of the C-N bonds.

We tried to fit data set 15 to $C c$ and obtained $R_{\mathrm{wp}} / R_{\text {exp }}=1.414$, which is significantly larger than the value $R_{\mathrm{wp}} / R_{\text {exp }}=1.384$ which we obtained for $C 2 / c$. In addition, the Reitveld refinement was unstable for our fit to $C c$. After a few iterations, $R_{\text {wp }}$ would reach a minimum value and then begin to increase, slowly at first, but eventually catastrophically. This occurred even when the refinement was heavily damped. Such an instability often indicates that there are too few restrictions on the assumed crystal symmetry. The instability did not occur in the refinement of the fit to $C 2 / c$.

Dultz et $a l .{ }^{4}$ did not attempt to fit their data to $C 2 / c$. They stated that $C 2 / c$ is "structurally improbable because the four carbon and nitrogen atoms in the unit cell would be in a special position in this case, which would not allow the physically true $\mathrm{C}-\mathrm{N}$ bond length of about $1.1 \AA$." From Table III we see that this is not the case. Also, they obtained a rather small vibrational amplitude for the $\mathrm{K}^{+}$ions (thermal parameter $B=0.2 \AA^{2}$, see Table III) and stated "that supports the absence of dynamic disorder in phase $C$ since large uncorrelated orientational jumps of the $\mathrm{CN}^{-}$ions would cause a relaxing of the $\mathrm{K}^{+}$ions and result in a considerable movement of these ions." In our fit, we obtained a much larger vibrational amplitude of the $\mathrm{K}^{+}$ions $\left(B=3.0 \AA^{2}\right.$, see Table III), supporting our conclusion that the $\mathrm{CN}^{-}$ions are indeed disordered.

Using the Landau theory of phase transitions in solids, the symmetry reduction in the phase transition $F m \overline{3} m \rightarrow C 2 / c$ is simpler to describe than that in $F m \overline{3} m \rightarrow C c$. Parlinski ${ }^{12}$ showed that for $F m \overline{3} m \rightarrow C c$, a coupled order parameter is necessary. In other words, the symmetry reduction $F m \overline{3} m \rightarrow C c$ cannot be accomplished with distortions described by a single irreducible representation (IR) of $F m \overline{3} m$. Two different IR's are necessary. On the other hand, the symmetry reduction $F m \overline{3} m \rightarrow C 2 / c$ requires only one IR ( $\hat{\tau}^{5}$ of star $k_{9}$, using the notation of Kovalev, ${ }^{13}$ or $L_{3}^{+}$, using the notation of Miller and Love ${ }^{14}$ ).

There are striking similarities between the phase diagrams of the various alkali cyanides. As the size of the alkali ion is increased, the phase lines move to lower pressures. (See, for example, Ref. 15.) In $\mathrm{RbCN}$, the transition to a monoclinic structure, like phase $C$ in $\mathrm{KCN}$, occurs at zero pressure. Using neutron diffraction, Rowe, Rush, and Lüty ${ }^{11}$ determined the space-group symmetry of that phase in $\mathrm{RbCN}$ to be $C c$, but with the $\mathrm{CN}^{-}$ions disordered with respect to $\mathrm{C}$ and $\mathrm{N}$, like our $C 2 / c$ structure. As the temperature is lowered, this orientational disorder in $\mathrm{RbCN}$ is frozen in place. This behavior is consistent with the dielectric measurements of Kondo, Schoemaker, and Lüty. ${ }^{16}$

The lattice parameters $a, b, c$ in $\mathrm{RbCN}$ are about $5 \%$ larger than those in $\mathrm{KCN}$ (due to the larger size of the $\mathrm{Rb}^{+}$ion), but the angle $\beta$ is nearly the same. We give in Table III the atomic positions for their structure of $\mathrm{RbCN}$. We see that their disordered $C c$ structure of $\mathrm{RbCN}$ is very similar to our $C 2 / c$ structure of $\mathrm{KCN}$, especially in the orientation of the $\mathrm{C}-\mathrm{N}$ bonds. 
Rowe et al. did not attempt to fit their data to $C 2 / c .^{17}$ Their primary interest was to show that the $\mathrm{CN}^{-}$ions are disordered. Besides, the peak shapes in their data were not well represented by the model in their refinement program, resulting in a rather poor value of $R_{\mathrm{wp}} / R_{\exp }$ (see Table III). Under these conditions, the difference in the fit to $C 2 / c$ and a disordered $C c$ may not have been significant enough to reach a conclusion anyway. We are suggesting that the structure of the monoclinic phase of $\mathrm{RbCN}$ may be $C 2 / c$ instead of $C c$.

We tried to fit our data to a disordered $C c$ structure. This is the same as the $C 2 / c$ structure, only with fewer restrictions on the atomic positions. We began with a structure identical to our $C 2 / c$ structure. Then we tried various small atomic displacements which would reduce the $C 2 / c$ symmetry to $C c$. Anything we tried only increased the value of $R_{\mathrm{wp}}$. We concluded that our $C 2 / c$ is stable with respect to such symmetry-breaking displacements.

Before leaving our discussion of phase $C$, we should comment about a possibly related phase, reported to be metastable, which exists between phases I and $A$ at ambient pressure. Cimino, Parry, and Ubbelohde ${ }^{18}$ discovered this phase, and Parry ${ }^{19}$ reported its structure to be monoclinic, space group $C c$. More recently, other monoclinic and triclinic structures have been reported for this phase. ${ }^{20,21}$ It has been suggested that this phase is actually the high-pressure phase $C$ which is stabilized by internal stresses in the multidomain crystal. However, in the $C c$ structure reported by Parry, the $\mathrm{CN}^{-}$ions are not aligned in the same direction as in phase $C$, and the size of the unit cell is different from phase $C$.

\section{PHASE $D$}

Phase $D$ is reported to be monoclinic, ${ }^{4}$ but its spacegroup symmetry and the positions of the atoms in the unit cell have not been previously determined. If phase $C$ has the disordered $C 2 / c$ structure, as discussed in the previous section, then we might expect the transition from phase $C$ to $D$ to be analogous to the transition from phase $A$ to $B$. The structure of phase $D$ should be like that of phase $C$, but with the $\mathrm{CN}^{-}$ions ordered with respect to $C$ and $N$. The resulting space-group symmetry should be No. $9 C c\left(C_{s}^{4}\right)$.

We tried fitting data set 16 to $C c$ and failed to obtain a satisfactory $R$ value. Phase $D$ is not just an ordered version of phase $C$. This is evident in the neutron-diffraction spectra shown in Fig. 3. The spectra for phases $A$ and $B$ are nearly identical. The ordering of the $\mathrm{CN}^{-}$ions does not have a large effect on the spectrum. On the other hand, the spectra for phases $C$ and $D$ show some striking differences. The transition from phase $C$ to $D$ involves something more than just an ordering of the $\mathrm{CN}^{-}$ ions.

When we found that we could not fit our data to $C c$, we removed the constraints of the symmetry of $C c$ and allowed the structure to have the complete freedom of space group No. $1 P 1\left(C_{1}^{1}\right)$. This allowed TOFLs to adjust the atomic positions more freely, and the refinement converged to a structure where all of the $\mathrm{CN}^{-}$ions lay nearly flat in parallel planes. This suggests the presence of mirror planes. If, in addition, the $\mathrm{CN}^{-}$ions are disordered with respect to $\mathrm{C}$ and $\mathrm{N}$ and centered on points of inversion, as in phase $C$, then we obtain a structure with the symmetry of space group No. $12 C 2 / m\left(C_{2 h}^{3}\right)$. The $\mathrm{K}^{+}$ions are at Wyckoff position $4 i(x, 0, z)$, and the $\mathrm{CN}^{-}$ions are centered at two different Wyckoff positions, $\mathrm{CN}(1)$ at $2 a(0,0,0)$ and $\mathrm{CN}(2)$ at $2 c\left(0,0, \frac{1}{2}\right)$.

We imposed the symmetry of $C 2 / m$ on the structure and obtained, from a fit of data set $16, R_{\mathrm{wp}} / R_{\exp }=$ 1.397 , nearly as good as our fit to $P 1$. (We discuss the possibility of triclinic symmetry below.) The details of the structure are given in Table IV. (We use the setting of unique axis $b$ and cell choice 1 in the International Tables for Crystallography. ${ }^{9}$ ) In the refinement, we constrained the C-N bond length to be $1.15 \AA$. In Fig. 3, we plot the difference between the data and the spectrum calculated from our best fit.

As in phase $C$, we found that in phase $D$ the broadening of diffraction peaks by inhomogeneous shear strains is very severe. We obtained the best fit to our data when the vectors $\hat{\mathbf{n}}_{1}$ and $\hat{\mathbf{n}}_{2}$, which describe the shear strain, are pointing in the directions $\mathbf{b}$ and $\mathbf{a}+0.17 \mathbf{c}$, respectively. This is slightly different from the directions in phase $C$.

The structure of phase $D$ is shown in Fig. 9. All of the $\mathrm{CN}^{-}$ions lie flat in mirror planes parallel to the plane of the figure. In the $C 2 / c$ structure of phase $C$ there are no mirror planes, only glide planes. We can see now why we had such difficulty in fitting our data in phase $D$ to $C c$. The space-group symmetry of phase $D$ is not a subgroup of that of phase $C$.

This large difference between the symmetries of phases $C$ and $D$ is in agreement with the $\mathrm{CN}^{-}$stretching frequencies measured by Raman scattering. ${ }^{15}$ In phase $C$, the two different $\mathrm{CN}^{-}$sites have identical local environments: same number of neighboring ions in the same relative directions (see Fig. 7). The $\mathrm{CN}^{-}$stretching frequency should be only slightly split. This appears as a single Raman line. In phase $D$, however, the two dif-

TABLE IV. Structure of phase $D$ fit to $C 2 / m$ (data set $16)$.

\begin{tabular}{lll}
\hline \hline$a$ & & $7.9402(4) \AA$ \\
$b$ & & $4.2694(1) \AA$ \\
$c$ & & $9.4758(4) \AA$ \\
$\beta$ & $x$ & $125.25(1)^{\circ}$ \\
$\mathrm{K}$ & $y$ & $0.531(1)$ \\
& $z$ & 0 \\
$B(\mathrm{~K})$ & & $0.749(1)$ \\
$\mathrm{C}(1), \mathrm{N}(1)$ & $x$ & $2.0(1) \AA^{2}$ \\
& $y$ & $-0.014(1)$ \\
$\mathrm{C}(2), \mathrm{N}(2)$ & $z$ & 0 \\
& $x$ & $0.053(1)$ \\
$B(\mathrm{C}, \mathrm{N})$ & $y$ & $0.087(1)$ \\
$R_{\mathrm{wp}} / R_{\exp }$ & $z$ & 0 \\
\hline \hline
\end{tabular}


Phase D

$\begin{array}{cccc}\otimes & \otimes & \otimes & \circledast \\ 0 & 0 & 8 & 0 \\ \otimes & \otimes & \otimes & \otimes \\ 8 & 0 & 0 & 0\end{array}$

FIG. 9. The structure of phase $D$. These are the same set of atoms shown in Fig. 4 for the cubic and orthorhomic phases. The plane of this figure is parallel to the monoclinic (010) plane, and the monoclinic $c$ axis points vertically upward on the page. The symbols in the figure have the same meaning as in Fig. 4.

ferent $\mathrm{CN}^{-}$sites have very different local environments (see Fig. 9). This causes a much greater splitting of the $\mathrm{CN}^{-}$stretching frequency, resulting in two resolved $\mathrm{Ra}-$ man lines.

We considered the possibility that the $\mathrm{CN}^{-}$ions are ordered in phase $D$. The space-group symmetry of an ordered phase would be a subgroup of $C 2 / \mathrm{m}$. We restricted our consideration to subgroups where the primitive unit cell is no more than double in size. From a table of isotropy subgroups, ${ }^{22}$ we find only three such subgroups which allow ordering of the $\mathrm{CN}^{-}$ions at both the Wyckoff $2 a$ and $2 c$ positions (see Table V). Since $\mathrm{CN}(1)$ at $2 a$ and $\mathrm{CN}(2)$ at $2 c$ are not related by symmetry, the ordering of the two different sets of $\mathrm{CN}^{-}$ions in the subgroups are independent of each other. We thus obtain for each subgroup two distinct ordered structures which differ by reversing the direction of $\mathrm{CN}(2)$.

As can be seen in Table $\mathrm{V}$, the disordered $C 2 / m$ structure is favored over $C m$ and $P 2_{1} / m$. However, $C 2 / m$ does not appear to be favored over $P \overline{1}$. We therefore examine the case of the $P \overline{1}$ structure a little more closely.

First, we note that in the ordered structures in Table $\mathrm{V}$, we assumed complete ordering of the $\mathrm{CN}^{-}$ions, i.e., $\mathrm{C}$ sites are occupied $100 \%$ of the time by $\mathrm{C}$ atoms, and $\mathrm{N}$ sites are occupied $100 \%$ of the time by $\mathrm{N}$ atoms. We were able to improve the fit to $P \overline{1}$ even further if we allowed partial ordering of the $\mathrm{CN}^{-}$ions. We obtained a best

TABLE V. Some possible structures of phase $D$ : the disordered $C 2 / m$ structure and six ordered structures which are subgroups of $C 2 / m$. For each subgroup, we give the irreducible representation (IR) of $C 2 / \mathrm{m}$ associated with the ordering [using the notation of Miller and Love (Ref. 14)]. Structures with the same IR differ by reversing the direction of $\mathrm{CN}(2)$. Each structure was fit to data set 16 using $m$ adjustable parameters, resulting in the values of $R_{\mathrm{wp}} / R_{\exp }$ shown.

\begin{tabular}{lllll}
\hline \hline Space group & IR & $\mathrm{CN}^{-}$ordering & $m$ & $\frac{R_{\mathrm{wp}}}{R_{\exp }}$ \\
\hline No. 12 C2/m( $\left(C_{2 h}^{3}\right)$ & & Disordered & 14 & 1.397 \\
No. 8 Cm $\left(C_{s}^{3}\right)$ & $\Gamma_{2}^{-}$ & Ferroelectric & 18 & 1.402 \\
No. 8 Cm $\left(C_{s}^{3}\right)$ & $\Gamma_{2}^{-}$ & Ferroelectric & 18 & 1.402 \\
No. 10 $P 2_{1} / m\left(C_{2 h}^{2}\right)$ & $Y_{2}^{-}$ & Antiferroelectric & 20 & 1.403 \\
No. 10 $P 2_{1} / m\left(C_{2 h}^{2}\right)$ & $Y_{2}^{-}$ & Antiferroelectric & 20 & 1.411 \\
No. 2 $P \overline{1}\left(C_{i}^{1}\right)$ & $V_{1}^{-}$ & Antiferroelectric & 27 & 1.329 \\
No. 2 $P \overline{1}\left(C_{i}^{1}\right)$ & $V_{1}^{-}$ & Antiferroelectric & 27 & 1.353 \\
\hline \hline
\end{tabular}

fit when the occupancy factor for $\mathrm{CN}(1)$ is $0.59(12)$ and for $\mathrm{CN}(2)$ is $0.58(13)$. Because of the large uncertainty in these numbers, this result is indistinguishable from complete disorder where the occupancy factor would be 0.5. The details of this $P \overline{1}$ structure are given in Table VI. We conclude that the good fit of the data to the $P \overline{1}$ structure is not an indication of $\mathrm{CN}^{-}$ordering. Our analysis indicates that in phase $D$ the $\mathrm{CN}^{-}$ions are most likely completely disordered.

To see why the data can be fit to $P \overline{1}$ better than to $C 2 / \mathrm{m}$, we must consider the effect of the lattice distortions allowed by triclinic symmetry. In $P \overline{1}$, the monoclinic $b$ axis is allowed to tilt away from a direction perpendicular to the monoclinic $a c$ plane. This distortion splits some of the monoclinic lines, causing them to appear broader. In our fit to $P \overline{1}$, the tilt of the $b$ axis was found to be $0.46(4)^{\circ}$, in a direction $-\mathbf{a}-0.16 \mathbf{c}$ (in terms of monoclinic basis vectors). This is a shear strain in nearly the same direction as the inhomogeneous shear strain which causes some of the lines to be broad. This triclinic distortion causes the very same lines to broaden. With the combination of the triclinic distortion and the inhomogeneous strain, we are able to model the shapes of these broadened lines better than with the inhomogeneous strain alone. This results in a better fit to $P \overline{1}$ than to $C 2 / m$.

We conclude that the inhomogeneous shear strain does not average to zero in our samples. This strain is centered about a nonzero tilt angle. The average symmetry of the crystal is not monoclinic, but triclinic.

This effect is amplified in sample 1 where the strains

TABLE VI. Structure of phase $D$ fit to $P \overline{1}$ (data set 16).

\begin{tabular}{lll}
\hline \hline$a$ & & $15.882(1) \AA$ \\
$b$ & & $4.493(1) \AA$ \\
$c$ & & $9.476(1) \AA$ \\
$\alpha$ & & $120.55(1)^{\circ}$ \\
$\beta$ & & $125.25(1)^{\circ}$ \\
$\gamma$ & $x$ & $28.37(3)^{\circ}$ \\
$\mathrm{K}(1)$ & $y$ & $-0.017(7)$ \\
& $z$ & $0.02(3)$ \\
$\mathrm{K}(2)$ & $x$ & $0.265(3)$ \\
& $y$ & $0.479(6)$ \\
$B(\mathrm{~K})$ & $z$ & $0.01(2)$ \\
$\mathrm{C}(1), \mathrm{N}(1)$ & & $0.236(3)$ \\
& $x$ & $2.0(2) \AA^{2}$ \\
$\mathrm{C}(2), \mathrm{N}(2)$ & $y$ & $0.234(1)$ \\
& $z$ & $0.040(3)$ \\
$\mathrm{C}(3), \mathrm{N}(3)$ & $x$ & $0.0525(2)$ \\
& $y$ & $0.267(1)$ \\
$\mathrm{C}(4), \mathrm{N}(4)$ & $z$ & $-0.046(3)$ \\
& $x$ & $-0.056(2)$ \\
$B(\mathrm{C}, \mathrm{N})$ & $y$ & $0.198(1)$ \\
$R_{\mathrm{wp}} / R_{\exp }$ & $z$ & $0.036(2)$ \\
\hline \hline & $x$ & $0.4473(2)$ \\
& $y$ & $0.298(1)$ \\
& $z$ & $-0.016(3)$ \\
& & $0.55(1)$ \\
& & $2.8(1) \AA^{2}$ \\
& & 1.318 \\
\hline
\end{tabular}


are larger. In Fig. 10, we show a portion of the neutrondiffraction spectra for phase $D$ in sample 2 , data set 16 (upper spectrum), and in sample 1, data set 9 (lower spectrum). In sample 1 we see that two of the monoclinic lines, $11 \overline{2}$ and 110 , appear to be each split into two lines. When we tried to fit data set 9 to $C 2 / m$, the best value of $R_{\mathrm{wp}} / R_{\text {exp }}$ we could obtain was 1.946 . Fitting the data to $P \overline{1}$, where the splitting of the lines is built into the model, we improved the value of $R_{\mathrm{wp}} / R_{\exp }$ to 1.648 . In this case, the tilt of the monoclinic $b$ axis was found to be $1.39(3)^{\circ}$, three times the tilt angle in sample 2 . On the other hand, the range of the inhomogeneous strain [proportional to the parameter $C$ in Eq. (2)] in sample 1 was found to be only about $23 \%$ greater than in sample 2. The severe line broadening in sample 1 is due more to an increase in the average strain than an increase in the range of strains.

There is one more issue to examine. If the symmetry of the crystal is some subgroup of $C 2 / m$, then we would expect to observe extra diffraction peaks not allowed by $C 2 / m$. For example, in phase $B$ we found two small peaks (see Fig. 5) which are not allowed by the symmetry of phase $A$. This is evidence that the symmetry of phase $B$ is some subgroup of the symmetry of phase $A$. We cannot find any "extra" peaks in our spectra for phase $D$. All of the observable peaks in phase $D$ can be labeled as allowed lines of the $C 2 / \mathrm{m}$ structure. The only evidence we can find for lower symmetry is the apparent splitting of some lines like those shown in Fig. 10.

We make the following conclusions about phase $D$ in KCN. First, the $\mathrm{CN}^{-}$ions are disordered with respect to $\mathrm{C}$ and N. Second, there is an inhomogeneous shear strain

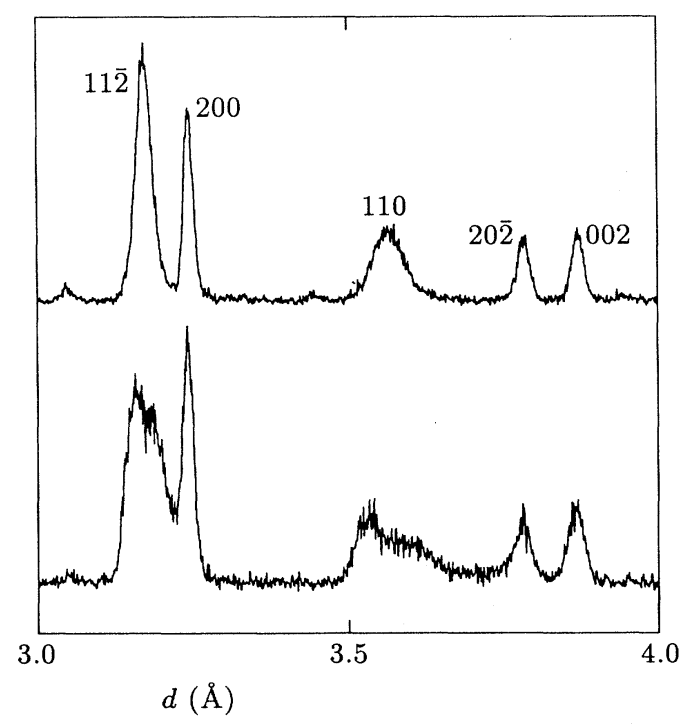

FIG. 10. A portion of the neutron-diffraction spectrum of phase $D$ in the two samples. The upper spectrum is from sample 2, data set 16 . The lower spectrum is from sample 1 , data set 9 . We label the five diffraction lines for phase $D$ in this portion of the spectrum. The small peaks, which are particularly evident in the upper curve, are from a small amount of phase $A$ present. which does not average to zero in our samples. The average symmetry is not monoclinic, but triclinic. However, these strains are likely caused by the domain structure in phase $D$. In a small single-domain crystal of phase $D$, we might expect to find the average symmetry to be exactly monoclinic with no triclinic distortion. Therefore, we prefer to call the symmetry of phase $D$ monoclinic, space group $C 2 / m$, with a small amount of triclinic distortion present in our samples.

Lastly, we can analyze the sequence of phase transitions $F m \overline{3} m \rightarrow C 2 / c \rightarrow C 2 / m$ (phases $\mathrm{I} \rightarrow C \rightarrow D$ ) using the Landau theory. $C 2 / c$ and $C 2 / m$ are both subgroups of $F m \overline{3} m$. They both arise from distortions belonging to the same irreducible representation (IR) of $F m \overline{3} m$ ( $\hat{\tau}^{5}$ of star $k_{9}$, using the notation of Kovalev, ${ }^{13}$ or $L_{3}^{+}$, using the notation of Miller and Love. ${ }^{14}$ ) This IR is eight-dimensional. The distortions are determined by the magnitude and direction of an eight-dimensional order parameter $\boldsymbol{\eta}$. In $F m \overline{3} m$, we have $\boldsymbol{\eta}=\mathbf{0}$ (no distortions). In $C 2 / c$, we have $\boldsymbol{\eta}=(a,-a, 0,0,0,0,0,0)$, and in $C 2 / m$, we have $\boldsymbol{\eta}=(a, a, 0,0,0,0,0,0)$. (Here we use the results of Stokes and Hatch. ${ }^{22}$ )

If we set all but the first two components of $\boldsymbol{\eta}$ to zero, the Landau free energy expanded out to sixth degree is

$$
\begin{aligned}
\Phi= & A\left(\eta_{1}^{2}+\eta_{2}^{2}\right)+B\left(\eta_{1}^{2}+\eta_{2}^{2}\right)^{2}+C\left(\eta_{1}^{2}+\eta_{2}^{2}\right)^{3} \\
& +D\left(11 \eta_{1}^{6}+15 \eta_{1}^{4} \eta_{2}^{2}+45 \eta_{1}^{2} \eta_{2}^{4}+9 \eta_{2}^{6}\right) \\
& +F\left(3 \eta_{1}^{5} \eta_{2}-10 \eta_{1}^{3} \eta_{2}^{3}+3 \eta_{1} \eta_{2}^{5}\right) .
\end{aligned}
$$

The difference in energy between $C 2 / c$ and $C 2 / m$ is due to the last term where $\eta_{2}$ appears with odd powers. When $F$ is positive, $C 2 / c$ is favored, and when $F$ is negative, $C 2 / m$ is favored. Since the transition $F m \overline{3} m \rightarrow C 2 / c$ is observed to be discontinuous, $B$ must be negative.

The sequence of phase transitions can thus be viewed in the following way. In phase $\mathrm{I}$, the value of $A$ is large and positive. The value of $A$ decreases with decreasing temperature, and when $A$ becomes small enough (but not quite zero), the transition to phase $C$ takes place. $F$ is positive at that point. As the temperature is lowered further, $F$ decreases until it becomes zero and then negative. As this point, the transition to phase $D$ takes place.

If the $\mathrm{CN}^{-}$ions are ordered in either phase $C$ or $D$, the space-group symmetry is some subgroup of $C 2 / c$ or $C 2 / m$, and the analysis by Landau theory becomes more complicated. Coupled order parameters belonging to more than one IR would be required.

\section{ACKNOWLEDGMENTS}

We are grateful for stimulating discussions with D. M. Hatch and K. J. Snow. We thank D. G. Hinks for providing us with one of the samples. We thank F. J. Rotella for assistance with the computer software used in analyzing the data. The work at Argonne National Laboratory was supported by the U.S. Department of Energy, BESMaterials Sciences, under Contract No. W-31-109-ENG38 . 


\section{APPENDIX: INHOMOGENEOUS STRAIN BROADENING}

A strain in a crystal changes the distance $d$ between planes of atoms and thus moves the position of a diffraction line. If the strain is inhomogeneous, then the distance $d$ between planes will be different in different regions of the crystal, giving rise to a distribution $\Delta d$ in the values of $d$ and thus broadening the diffraction lines. We derive here an expression for $\Delta d$ in terms of the inhomogeneous distribution $\Delta \epsilon_{i j}$ of the components of the strain tensor.

The value of $d$ for a diffraction line $(h k l)$ is given by

$$
d=1 / G \text {, }
$$

where $G$ is the magnitude of the reciprocal lattice vector,

$$
\mathbf{G}=h_{i} \mathbf{b}_{i},
$$

and $\mathbf{b}_{i}$ are the basis vectors of the reciprocal lattice. The coefficients $h_{i}$ are the Miller indices $h, k, l$. We define a strain in terms of its effect on three mutually perpendicular unit vectors $\mathbf{e}_{i}$. The strain results in the vectors (to first order),

$$
\mathbf{e}_{i}^{\prime}=\left(\delta_{i j}+\epsilon_{i j}\right) \mathbf{e}_{j},
$$

where $\delta_{i j}$ is the Kronecker delta function and $\epsilon_{i j}$ are components of a strain tensor, chosen to be symmetric, i.e., $\epsilon_{j i}=\epsilon_{i j}$. Note that in general the strain affects both the magnitude and direction of $\mathbf{e}_{i}^{\prime}$ so that the vectors $\mathbf{e}_{i}^{\prime}$ are not unit vectors and are not mutually perpendicular.

We write the basis vectors $\mathbf{a}_{i}$ of the direct lattice in the form

$$
\mathbf{a}_{i}=a_{i j} \mathbf{e}_{j} .
$$

The strain produces basis vectors,

$$
\mathbf{a}_{i}^{\prime}=a_{i j} \mathbf{e}_{j}^{\prime}=a_{i j}\left(\delta_{j k}+\epsilon_{j k}\right) \mathbf{e}_{k} .
$$

The basis vector $b_{1}$ is given by

$$
\mathbf{b}_{1}=\frac{1}{V}\left(\mathbf{a}_{2} \times \mathbf{a}_{3}\right)
$$

where $V$ is the volume of the unit cell of the direct lattice. Similar expressions can be obtained for $\mathbf{b}_{2}$ and $\mathbf{b}_{3}$ by cyclic permutations of the indices in the above expression. Similar to Eq. (A4), we also write these vectors in the form

$$
\mathbf{b}_{i}=b_{i j} \mathbf{e}_{j}
$$

The strain produces basis vectors,

$$
\mathbf{b}_{1}^{\prime}=\frac{1}{V^{\prime}}\left(\mathbf{a}_{2}^{\prime} \times \mathbf{a}_{3}^{\prime}\right)
$$

Substituting the expression for $\mathbf{a}_{i}^{\prime}$ from Eq. (A5) into this equation and after some algebraic manipulation, keeping only terms which are first order in $\epsilon_{i j}$, we obtain

$$
\mathbf{b}_{i}^{\prime}=b_{i j}\left(\delta_{j k}-\epsilon_{j k}\right) \mathbf{e}_{k} .
$$

We note that this expression is similar to Eq. (A5). Only the minus sign is different. If we now write $\mathbf{G}$ in the form

$$
\mathbf{G}=G_{i} \mathbf{e}_{i},
$$

we obtain

$$
\mathbf{G}^{\prime}=G_{i}\left(\delta_{i j}-\epsilon_{i j}\right) \mathbf{e}_{j}
$$

The value of $d$ after the strain is given by

$$
d^{\prime}=1 / G^{\prime} \text {. }
$$

Expanding this expression to first order in $\epsilon_{i j}$, we obtain

$$
d^{\prime}=d\left(1+G_{i} \epsilon_{i j} G_{j} / G^{2}\right) \text {. }
$$

Recognizing that $G_{i} / G=\cos \phi_{i}$, where $\phi_{i}$ is the angle between $\mathbf{G}$ and $\mathbf{e}_{i}$, we write

$$
d^{\prime}=d\left(1+\epsilon_{i j} \cos \phi_{i} \cos \phi_{j}\right) .
$$

If the strain is inhomogeneous, there is a spread $\Delta \epsilon_{i j}$ in the values of $\epsilon_{i j}$ and a resulting spread in the values of $d^{\prime}$ given by

$$
\Delta d=d \Delta \epsilon_{i j} \cos \phi_{i} \cos \phi_{j},
$$

or, when written out explicitly,

$$
\begin{aligned}
\Delta d=d & \left(\Delta \epsilon_{11} \cos ^{2} \phi_{1}+\Delta \epsilon_{22} \cos ^{2} \phi_{2}+\Delta \epsilon_{33} \cos ^{2} \phi_{3}\right. \\
& +2 \Delta \epsilon_{12} \cos \phi_{1} \cos \phi_{2}+2 \Delta \epsilon_{13} \cos \phi_{1} \cos \phi_{3} \\
& \left.+2 \Delta \epsilon_{23} \cos \phi_{2} \cos \phi_{3}\right) .
\end{aligned}
$$

For the case of the pure shear strain in $\mathrm{KCN}$, only one off-diagonal element $\epsilon_{12}$ of the strain tensor is nonzero (if we choose an appropriate coordinate system), and we obtain

$\Delta d=2 d \Delta \epsilon_{12} \cos \phi_{1} \cos \phi_{2}=C d \cos \phi_{1} \cos \phi_{2}$,

where $C$ is an adjustable parameter to be determined by the least-squares process in TOFLS.

For a more general form of inhomogeneous strain, we can obtain a useful expression by combining Eqs. (A1), (A2), (A7), and (A13), and we obtain

$$
\Delta d=d^{3} h_{k} b_{k i} \Delta \epsilon_{i j} h_{l} b_{l j},
$$

which is of the form

$\Delta d_{h k l}=d^{3}\left(C_{1} h^{2}+C_{2} k^{2}+C_{3} l^{2}+2 C_{4} h k+2 C_{5} h l+2 C_{6} k l\right)$. 
${ }^{1}$ J. M. Bijvoet and J. A. Lely, Rec. Trav. Chim. Pays-Bas 59, 908 (1940).

${ }^{2}$ D. L. Decker, R. A. Beyerlein, G. Roult, and T. G. Worlton, Phys. Rev. B 10, 3584 (1974).

${ }^{3}$ J. M. Rowe, J. J. Rush, and E. Prince, J. Chem. Phys. 66, 5147 (1977).

${ }^{4}$ W. Dultz, H. H. Otto, H. Krause, and J. L. Buevoz, Phys. Rev. B 24, 1287 (1981).

${ }^{5}$ J. D. Jorgensen, J. Faber Jr., J. M. Carpenter, R. K. Crawford, J. R. Haumann, R. L. Hitterman, R. Kleb, G. E. Ostrowski, F. J. Rotella, and T. G. Worlton, J. Appl. Cryst. 22, 321 (1989).

${ }^{6}$ J. D. Jorgensen, S. Pei, P. Lightfoot, D. G. Hinks, B. W. Veal, B. Dabrowski, A. P. Paulikas, R. Kleb, and I. D. Brown, Physica C 171, 93 (1990).

${ }^{7}$ R. B. Von Dreele, J. D. Jorgensen, and C. G. Windsor, J. Appl. Cryst. 15, 581 (1982).

${ }^{8}$ J. M. Rowe, D. G. Hinks, D. L. Price, S. Susman, and J. J. Rush, J. Chem. Phys. 58, 2039 (1973).

${ }^{9}$ International Tables for Crystallography, edited by T. Hahn (Reidel, Dordrecht, 1983), Vol. A.

${ }^{10}$ H. T. Stokes and D. M. Hatch, Phys. Rev. B 30, 3845 (1984).
${ }^{11}$ J. M. Rowe, J. J. Rush, and F. Lüty, Phys. Rev. B 29, 2168 (1984).

${ }^{12}$ K. Parlinski, Z. Phys. B 56, 51 (1984).

${ }^{13}$ O. V. Kovalev, Irreducible Representations of the Space Groups (Gordon and Breach, New York, 1965).

${ }^{14}$ S. C. Miller and W. F. Love, Tables of Irreducible Representations of Space Groups and Co-Representations of Magnetic Space Groups (Pruett, Boulder, 1967); A. P. Cracknell, B. L. Davies, S. C. Miller, and W. F. Love, Kronecker Product Tables (Plenum, New York, 1979), Vol. 1.

${ }^{15}$ W. Dultz and H. Krause, Phys. Rev. B 18, 394 (1978).

${ }^{16}$ Y. Kondo, D. Schoemaker, and F. Lüty, Phys. Rev. B 19, 4210 (1979).

${ }^{17} \mathrm{~J}$. M. Rowe (private communication).

${ }^{18}$ A. Cimino, G. S. Parry, and A. R. Ubbelohde, Proc. R. Soc. London, Ser. A 252, 445 (1959).

${ }^{19}$ G. S. Parry, Acta Crystallogr. 15, 601 (1962).

${ }^{20}$ Y. Yoshimura and K. Shimaoka, J. Phys. Soc. Jpn. 57, 3052 (1988).

${ }^{21}$ Y. Yoshimura, J. Phys. Soc. Jpn. 58, 3993 (1989).

${ }^{22} \mathrm{H}$. T. Stokes and D. M. Hatch, Isotropy Subgroups of the 230 Crystallographic Space Groups (World Scientific, Singapore, 1988). 